\title{
Spontaneous Wilms Tumor Rupture: A Preoperative Clinical Diagnosis
}

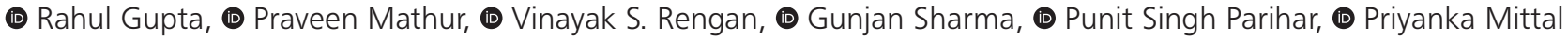 \\ Department of Paediatric Surgery Sawai Man Singh Medical College, Jaipur, Rajasthan, India
}

\begin{abstract}
We report a rare case of a spontaneous Wilms tumor rupture in a 13-month-old male who presented with a rapidly increasing abdominal distension. The child had acute exacerbation of symptoms which precipitated an emergency left radical nephrectomy and renal venectomy (due to densely adherent tumoral embolus in left renal vein and Inferior Vena Cava) indicated for intraperitoneal tumor rupture. Postoperative histopathology confirmed a Wilms tumor; classified as stage IIIc. He is on a three-drug chemotherapy and doing well on follow-up. Preoperative spontaneous Wilms tumor rupture is a clinico-radiological challenge. A high index of suspicion is a prerequisite for diagnosis. However, an upfront emergency radical nephrectomy should be discussed.

Keywords: Emergency, preoperative, radical nephrectomy, rupture, spontaneous, Wilms tumor
\end{abstract}

\section{Introduction}

Wilms tumor rupture is rare, constituting $3 \%$ of all the cases of Wilms tumor $(1,2)$. Preoperative rupture is rarer and could either be post-traumatic or spontaneous in nature $(1,2)$. Diagnosis of preoperative rupture is based on clinico-radiological signs $(1,3)$. Confirmation is either intraoperative and subsequently on histopathological evaluation $(1,4,5)$. We report a very rare case of spontaneous Wilms tumor rupture in a child who was diagnosed clinically and then confirmed intraoperatively (6). A mini review of preoperative Wilms tumor rupture is contemplated.

\section{Case Presentation}

A thirteen-month-old male, weighing $8,200 \mathrm{~g}$, second in birth order, and a product of non-consanguineous marriage presented with a rapidly increasing abdominal distension evolving for the last two months. There were episodes of low-grade fever, loss of appetite, and crying episodes for similar duration. On examination, the child was conscious, hemodynamically stable, but in agony due to pain. He was pale, a pulse rate of $116 / \mathrm{min}$, respiratory rate of $36 / \mathrm{min}$ with mild-to-moderate dyspnea. Perabdominal examination revealed a firm-to-hard, large, tender lump occupying left half of abdomen and reaching the midline; genitalia were normal.

Baseline blood investigations (inv.) revealed anemia (hemoglobin-6 g/dL), and leukocytosis (total leucocyte

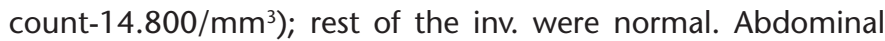
ultrasonography (USG) confirmed a large $(13 \times 11 \mathrm{~cm})$, heterogeneous, solid-cystic left kidney tumor with vascularity (on color Doppler) and tumor thrombus extending in left renal vein $(19 \mathrm{~mm})$.

Patient was optimized; three-packed RBC transfusions were administered. Patient was planned for further evaluation with preoperative abdominal contrast-enhanced computerized tomography, fine needle aspiration cytology, and neo-adjuvant chemotherapy. However, the child had acute exacerbation of pain with marked abdominal distension. A moderate respiratory distress and nasal flaring which was suggestive of splinting of diaphragm was also observed. On palpation, there was a sudden increase in size of lump, marked tenderness and abdominal wall induration on the left half of the abdomen, suggestive of tumor rupture necessitating emergency laparotomy. The patient was transferred to the operation room after obtaining informed written consent from the parents.

Exploratory laparotomy revealed a large ruptured left renal tumor causing antero-medial displacement and compression of the descending colon as shown in the diagrammatic representation (Figure 1). The tumor had ruptured (eroded) intra-peritoneally through a large, $4 \times 4 \mathrm{~cm}$ size rent in the descending mesocolon (Figure 2) with tumor fragments, necrotic material and hemorrhagic fluid in the pelvic cavity and Morrison's pouch. Dissection of the tumor from the renal fossa 


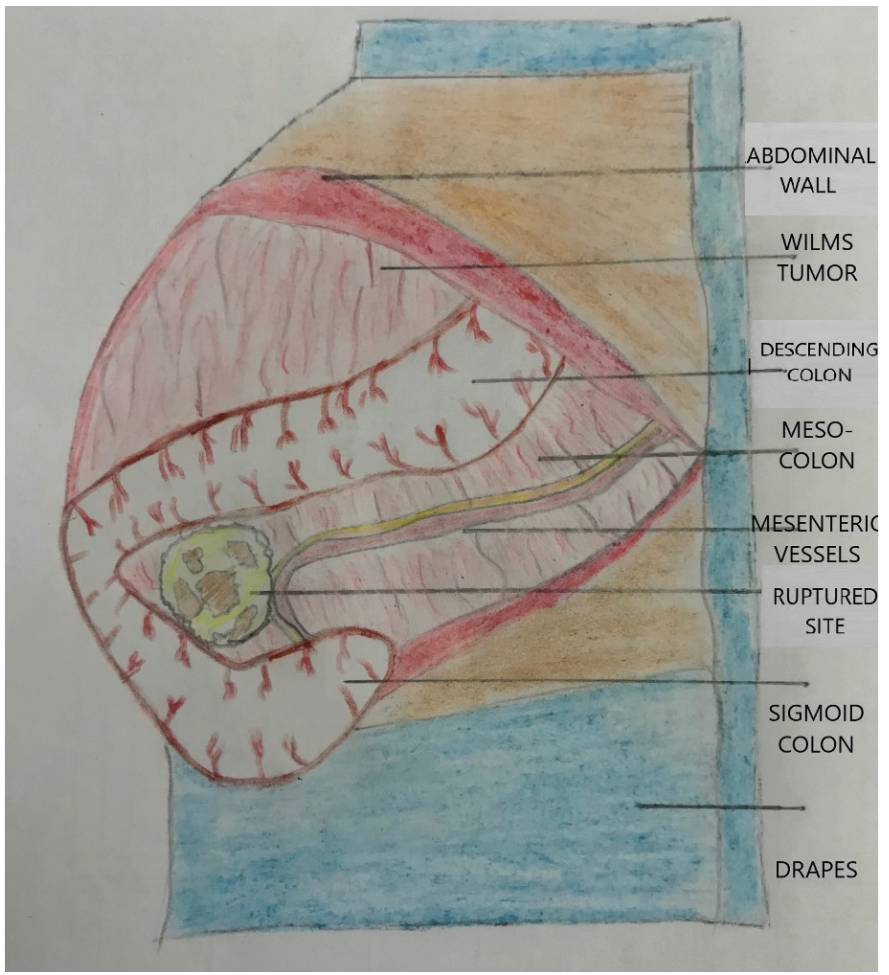

Figure 1. Diagrammatic representation (by RG) of a large left renal tumor causing antero-medial displacement of the descending colon; tumor is ruptured intraperitoneally through a large, $4 \times 4 \mathrm{~cm}$ size rent in the descending mesocolon with tumor fragments and necrotic material seen coming out of the surface

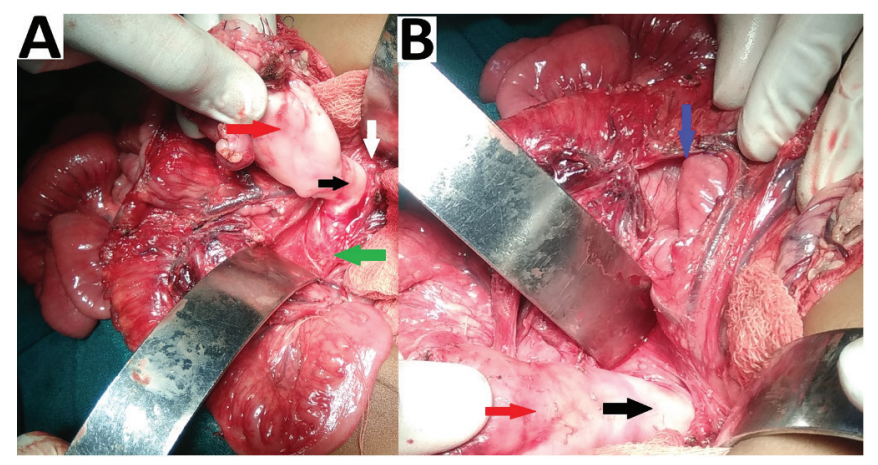

Figure 2. Intraoperative photographs showing (A) tumor embolus (red arrow) extending into the left renal vein (black arrow) and IVC (green arrow); the tumor embolus is extending into the IVC superiorly (white arrow); (B) a large, $4 \times 4 \mathrm{~cm}$ size rent in the descending mesocolon (blue arrow) through which the tumor had ruptured (eroded) intra-peritoneally

IVC: Inferior Vena Cava

and adherent mesocolon was performed. The tumor extended into the left renal vein and Inferior Vena Cava (IVC) (Figure 2). Radical left-sided nephrectomy (Figure 3) with the removal of para-aortic and aorto-caval lymph nodes was performed. Left renal venotomy was performed to remove the densely adherent tumor embolus in the renal vein and IVC, which was unsuccessful. Renal venectomy was performed at its junction with the IVC (Figure 4). Postoperative recovery was satisfactory, except for a few episodes of fever spikes.

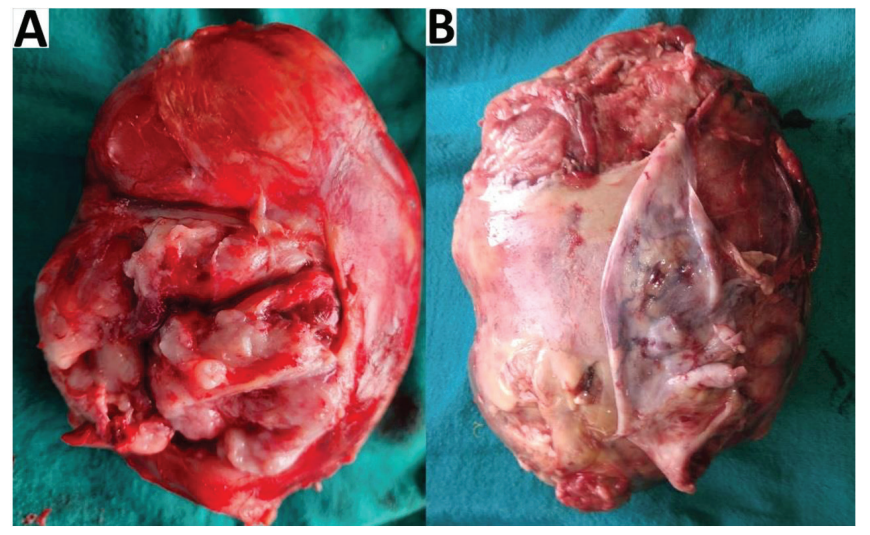

Figure 3. Radical nephrectomy specimen showing (A) peritoneal surface with ruptured area; (B) retroperitoneal surface

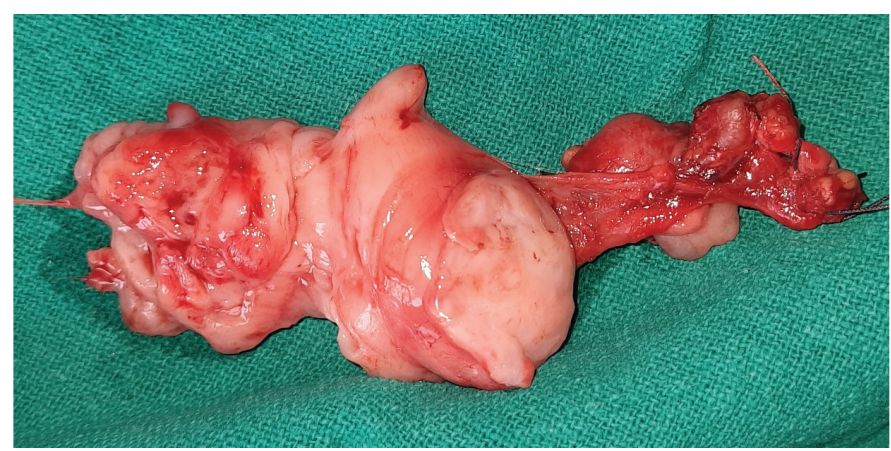

Figure 4. Renal venectomy specimen with densely adherent tumor embolus

Histopathology confirmed the diagnosis of triphasic nephroblastoma (Wilms tumor) with favorable histology; tumor embolus in the renal vein. There were no tumor cells in lymph nodes samples. The pathologist confirmed the rupture of the tumor. Pediatric oncology opinion was sought; the stage of the tumor was classified as Illc on the basis of the radiological, intraoperative findings, and pathological evaluation. The patient is on 3 drug chemotherapy treatment protocol/regimen [recommended as per International Society of Pediatric Oncology (SIOP) 2001] with us and is under 3 months follow-up.

\section{Discussion}

Wilms tumor constitutes $6 \%$ of all pediatric malignant tumors (5). The most affected age group is 2-3 years, though it may present in early infancy to 10 years and beyond in the adults $(7,8)$. Wilms tumor is a rapidly growing tumor, and usually attains an enormous size before it is diagnosed (9). Also, its doubles after 11 days, making it prone to rupture, either preoperatively or intraoperatively (spill) (9). Tumor rupture is a potentially significant event triggering tumor dissemination, both locally (through lymphatics) and remotely (through blood stream) (2). Tumor spillage is considered if there is preoperative tumor rupture, intraoperative tumor spill, or a tumor biopsy. Intraoperative tumor spill and tumor biopsy are intervention-related events (3). The SIOP Nephroblastoma Trial Group recommends neo-adjuvant chemotherapy for prevention of tumor rupture (10). In our case, the tumor ruptured before chemotherapy was initiated. 
Tumors greater than $12 \mathrm{~cm}$ in size have a two-fold risk of intraoperative rupture than smaller lumps (11). Intraperitoneal rupture (our case) is less common than retroperitoneal type $(4,5)$. Rupture is common in males than females; right side is greater than left side $(4,5)$.

Preoperative Wilms tumor rupture could either be posttraumatic or spontaneous in type. Spontaneous ruptures (as appreciated in our case) are rarer than the former $(1,2)$. In a large series of 1853 Wilms tumors, preoperative rupture was appreciated in $5 \%(88)$ of patients (1). Preoperative rupture ranged from $2.1 \%$ to $23 \%$ in different studies $(4,12)$. A study in Europe reported it to be $3 \%$ Wilms tumor (1). During a 9 year period from 2012 to 2020, 152 patients with Wilms tumor were surgically managed in our institute. Among these, this is the first (index) case of preoperative spontaneous Wilms tumor rupture. It is due to delay in seeking treatment, large tumor size $(13 \mathrm{~cm})$, rapid growth with involvement of renal vein and IVC by densely adherent large tumor thrombus (Figure 2 and Figure 3) (by senior author, RG).

Consequences of preoperative Wilms tumor rupture are: upstaging (IIIC), tumor spillage, surgery without neo-adjuvant chemotherapy, alteration in treatment (intensive chemotherapy with abdominal radiotherapy), and altered prognosis with adverse effects due to tumor dissemination $(4,5,13)$. Wilms tumor spillage increases the risk of abdominal recurrence to 20\% (14).

Clinical signs suggestive of preoperative Wilms tumor rupture include: acute exacerbation of abdominal pain, history of trauma, retroperitoneal or intraperitoneal hemorrhage, and sudden drop in $\mathrm{Hb}$ requiring blood transfusion (5).

Radiological signs [computerized tomography (CT) or USG findings] suggestive of preoperative rupture are: (a) poorly circumscribed mass with a non-delineated mass (b) fat stranding around tumor with linear areas of soft-tissue attenuation in peritumoral fat suggestive of capsular breech, (c) retroperitoneal hemorrhage or fluid in the sub-capsular (crescent-shaped fluid collection following the contour of the kidney), perirenal, or pararenal space, (d) hemorrhagic ascites or ascites beyond the cul-de-sac, (e) peritoneal implants, (f) retroperitoneal tumor nodules separate from primary tumor (g) mesenteric infiltration (h) ipsilateral pleural effusion, (i) tumoral fracture communicating with peritoneal effusion and (j) intra-tumoral hemorrhage. CT has high specificity but relatively low sensitivity in the detection of preoperative Wilms tumor rupture $(1,3,4,5)$. The most consistent CT sign is the presence of ascites beyond the cul-de-sac, irrespective of attenuation (3).

Management of preoperative Wilms tumor rupture is divided into either immediate or delayed group on the basis of timing of radical nephrectomy (5). Emergency surgery for preoperative Wilms tumor rupture range from $1.8 \%$ and $3 \%$ (4). The decision is based on clinico-radiological findings $(1,3,4,5)$. In the immediate group, upfront surgery is performed, followed by adjuvant chemotherapy. In the delayed group, upfront chemotherapy followed by surgical intervention is achieved $(5,10)$. The criteria for immediate surgery include localized rupture, completely resectable forms, tumor not crossing the midline and without IVC thrombus. Delayed surgery after preoperative chemotherapy has been recommended with non-localized rupture, large tumors that cannot be completely resected, tumors infiltrating to surrounding organs and long IVC tumor thrombus $(5,10)$.

They were more cases of metastasis and recurrence in immediate group (2/13) than delayed group (8/28) in a large series of Wilms tumor, though not significant (41) (5). Survival outcomes were better in immediate than in the delayed group $(5,14)$.

\section{Conclusion}

Preoperative spontaneous Wilms tumor rupture is a clinicoradiological challenge. A high index of suspicion is a prerequisite for diagnosis. Therefore, an upfront emergency radical nephrectomy should be discussed.

\section{Acknowledgements}

Publication: The results of the study were not published in full or in part in form of abstracts.

Contribution: I am sincerely thankful to faculty, residents and nursing staff of department of Paediatric Surgery, SMS Medical College, Jaipur, for helping us in this endeavor.

Conflict of Interest: No conflict of interest was declared by the authors.

Financial Disclosure: The authors declared that this study received no financial support.

\section{Ethics}

Informed Consent: The patient was transferred to the operation room after obtaining informed written consent from the parents.

Peer-review: Externally peer-reviewed.

\section{Authorship Contributions}

Supervision: R.G., Concept: R.G., Design: R.G., Data Collection or Processing: R.G., V.S.R., G.S., P.S.P., Mi., Analysis or Interpretation: P.M., Literature Search: R.G., V.S.R., G.S., Critical Review: P.M., Writing: R.G., V.S.R., G.S., P.S.P., Mi.

\section{References}

1. Leape LL, Breslow NE, Bishop HC. The surgical treatment of Wilms' tumor: results of the National Wilms' Tumor Study. Ann Surg 1978; 187:351-356.

2. Apoznanski W, Patkowski D, Polok M, et al. Preoperative Wilms tumor rupture: Controversial diagnosis. Case report. Pediatr Pol 2017;92:786-788.

3. Khanna G, Naranjo A, Hoffer F, et al. Detection of preoperative Wilms tumor rupture with CT: a report from the Children's Oncology Group. Radiology 2013;266:610-617.

4. Brisse HJ, Schleiermacher G, Sarnacki S, et al. Preoperative Wilms tumor rupture. A retrospective study of 57 patients. Cancer 2008; 113:202-213.

5. Zhang $Y$, Song HC, Yang YF, et al. Preoperative Wilms tumor rupture in children. Int Urol Nephrol 2021;53:619-625.

6. Godzinski J, Weirich A, Tournade MF, et al. Primary nephrectomy for emergency: a rare event in the International Society of Paediatric Oncology Nephroblastoma Trial and Study no. 9. Eur J Pediatr Surg 2001;11:36-39.

7. Nakamura L, Ritchey M. Current management of Wilms' tumor. Curr Urol Rep 2010;11:58-65. 
8. Heyns CF, Rossouw DJ. Spontaneous rupture of adult Wilms' tumor. Cancer 1989;64:173-177.

9. Zoubek A, Slavc I, Mann G, et al. Natural course of a Wilms' tumour. Lancet 1999;354:344.

10. Mitchell C, Pritchard-Jones $\mathrm{K}$, et al. Immediate nephrectomy versus preoperative chemotherapy in the management of non-metastatic Wilms' tumour: results of a randomised trial (UKW3) by the UK Children's Cancer Study Group. Eur J Cancer 2006;42:2554-2562.

11. Gow KW, Barnhart DC, Hamilton TE, et al. Primary nephrectomy and intraoperative tumor spill: report from the Children's Oncology Group (COG) Renal Tumors Committee. J Pediatr Surg 2013;48:34-38
12. Adu J, Watson T. Imaging features of preoperative Wilms tumour rupture on CT and MRI with histopathological confirmation. Arch Dis Child 2018;103:A43.

13. Le Rouzic MA, Mansuy L, Galloy MA, et al. Agreement between clinicoradiological signs at diagnosis and radiohistological analysis after neoadjuvant chemotherapy of suspected Wilms tumor rupture: consequences on therapeutic choices. Pediatr Blood Cancer 2019;66:e27674.

14. Burgers JM, Tournade MF, Bey P, et al. Abdominal recurrences in Wilms' tumours: a report from the SIOP Wilms' tumour trials and studies. Radiother Oncol 1986;5:175-182. 